\title{
State estimation-based target tracking and applications of multi sensor data fusion
}

\section{David Kondru* and Mehmet Celenk}

\author{
School of EECS, \\ Ohio University, \\ Athens, OH, 45701, USA \\ Email: k.rajusolomon@gmail.com \\ Email: celenk@ohio.edu \\ *Corresponding author
}

\section{Xiaoping A. Shen}

Department of Mathematics,

Ohio University, Athens, OH, 45701, USA

Email: shenx@ohio.edu

\begin{abstract}
Detection, discrimination and tracking of a target under a given dynamic environment is one of the main challenges of an integrated sensory system. A combination of two or more sensors will always provide a better position estimate rather than a single sensor. The advantages of multi-sensor data fusion over a conventional single sensor tracking are presented in this paper. Using state estimators from simple linear rustic filters to a complex nonlinear filter, the tracking of target performing three different motions with sensor noises are presented in this paper. RADAR and the infrared search and track (IRST) are the two sensors considered based on which a complete mathematical modelling and simulation of the sensor measurements and tracking methodologies are utilised. The extension of the paper also presents the image fusion techniques using a widely known technique known as principle component analysis method. The RMS errors in position as well as image error measurements are performed that shows the superiority of the multi sensor data fusion process.
\end{abstract}

Keywords: Kalman filter; particle filter; measurement fusion; state vector fusion; SVF; image fusion; principle component analysis.

Reference to this paper should be made as follows: Kondru, D., Celenk, M. and Shen, X.A. (2019) 'State estimation-based target tracking and applications of multi sensor data fusion', Int. J. Forensic Software Engineering, Vol. 1, No. 1, pp.32-46.

Biographical notes: David Kondru is a graduate student in Ohio University where he is pursuing Electrical Engineering. Currently, he works as a Teaching and Research Assistant in School of EECS. His research area includes signal and image processing, and computer vision.

Mehmet Celenk received his BS and MS from Istanbul Technical University, in 1974 and 1976, in Electrical and Communications Engineering, and $\mathrm{PhD}$ from 
Stevens Institute of Technology in Electrical Engineering and Computer Science (EECS), in 1983, where he was the Robert Crook Stanley Graduate Fellow in 1985. He served on the Turkish Army in 1984-1985 as a Lieutenant and joined Ohio University (OU) in 1985, where he is currently a Professor of the School of EECS. He has published 300 articles, received $\$ 600 \mathrm{~K}$ hypercube processor grant, participated in $\$ 450 \mathrm{~K}$ Tubitak Autonomous Vehicle Design and Development grant, and secured $\$ 120 \mathrm{~K}$ fund for visiting scholars' R\&D projects. He directed his $35 \mathrm{MS} / \mathrm{PhD}$ theses/dissertations in the School of EECS of OU.

Xiaoping A. Shen received her $\mathrm{PhD}$ in Applied Mathematics from the University of Wisconsin, WI, USA. She was a Post-Doctoral Fellow of the National Science Foundation and the Office of Naval Research, a Summer Faculty Research Fellow of the Air Force Office of Scientific Research. He is currently a Professor of Applied Mathematics at Ohio University, Athens, $\mathrm{OH}$, USA. Her research interests are extended over areas of computational harmonic analysis (wavelets), approximation theory, and nonparametric statistics with applications in the area of signal/image processing, information theory, and data processing.

This paper is a revised and expanded version of a paper entitled 'Performance evaluation of state estimators for airborne target tracking using multi sensor data fusion', presented at 2018 IEEE Electro-Information Technology (EIT), Oakland University, Rochester, MI, 3-5 May 2018.

\section{Introduction}

A high degree of importance has been given to detection and tracking of targets for any aerospace guidance and control systems. For civilian sensors such as primary surveillance radar (PSR) and secondary surveillance radar (SSR), the tracking of incoming and departing airplanes is of utmost importance. In case of military sensors, there are sequences of successful tasks such as detection, discrimination, tracking and interception has to be done at high degree of accuracy (Mahafza, 2000). Out of many sensors, a digital processing technique such as pulse compressed-based phased array radars were introduced to achieve those tasks and one such an application is moving target indicating (MTI) radar (Minvielle, 2005). The targets information such as its relative range, azimuth and elevation, as well as its velocity is the primary sensor data necessary for tracking. Based on the type of scenario different tracking methodologies can be employed with different sensor technologies (Rodríguez-Canosa et al., 2014; Khalifa, 2013; Landsat 8, 2013). For instance, network centric warfare is one of the key components of Department of Defence for the US forces to enhance the situational awareness in the battle space (Friedman, 2009). Also, the positioning and the installation of sensors vary on application of detection and tracking methods (Dimitrios, 2007) the purpose of which is to enhance the dominance of sensor fusion over single sensor tracking. Optical detection and tracking using optical and infrared cameras and thermal cameras are advancing in today's vision-based applications even for targets having very less signature (Fabrizio, 2014). Many new modern technologies are developing sophisticated detection and tracking methods for better awareness and perception. One such as example is FLIR-based Argus 
and Cerebrus integrated surveillance systems (FLIR the World's Sixth Sense, 2016; CommandSpace Cereberus Datasheet, 2018). Nevertheless, irrespective of any modern technology the target track has to follow a closed loop system tracker due to inherent internal noises and external noises. Although the radar is an active sensor, it has a better resolution in range besides its poor resolution in angular measurements. But, where as, the infrared search and track (IRST) sensors being passive systems has good resolution in angular measurements. On the other hand a combination of one or more optical sensors and their fusion could also enhance the performance of optical detection and tracking. Utilising the fusion of same sensor configuration or different configuration is based on the fact that the entire target data may not be obtained with a single sensor, however, regardless of their inherent characteristics, the fusion process will always provide a better estimate of target information. With these insights Section 2 presents the mathematical models for the target and the sensor dynamics. Section 3 presents the state estimation methodology and the filtering algorithms. Section 4 explains the type of sensor fusion for radar and IRST sensors. Section 5 presents the results of the first version of this paper. Section 6 presents the PCA-based image fusion-based and the experimental analysis with image error measurements.

\section{Geometric relationship between sensors and target}

By assuming the location of the sensors on the same platform with a negligible distance and using flat earth approximation (Garwin, 1999) the geometric relationship between the sensor and the target is represented in a three-dimensional spherical coordinate system shown in Figure 1.

Figure 1 3D spherical coordinate system

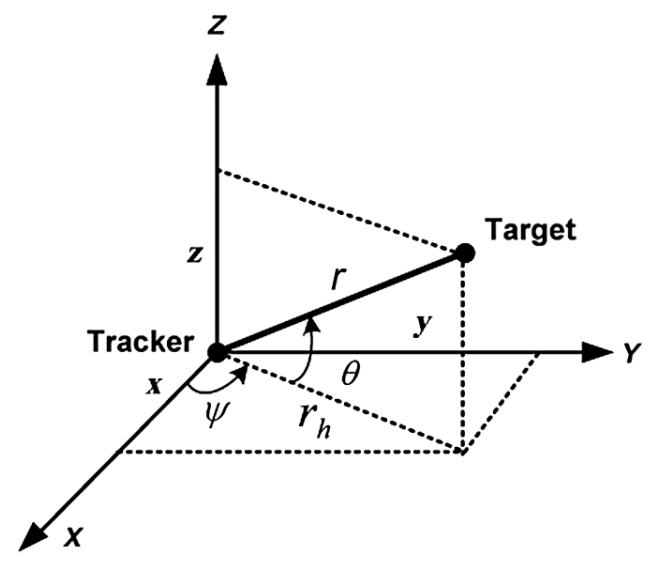

Source: Nykamp DQ

A combination of RADAR and FLIR which provides the targets relative range $(r)$, azimuth $(\varphi)$ and elevation $(\theta)$ can be obtained by considering different target motions (Lesson 2 - Projectile Motion; Weisstein; Gordon et al., 1993). A three dimensional representation of targets position, velocity and acceleration is shown in equation (1). 


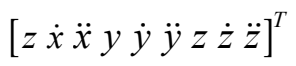

The mathematical notation of the LOS system measurements measured by class of LOS systems such as airborne RADAR, FLIR, TV, laser ranging, etc. (Grewal and Andrews, 2001) are given by:

$$
\begin{aligned}
& r=\sqrt{\Delta x^{2}+\Delta y^{2}+\Delta z^{2}} \\
& \psi=\tan ^{-1}\left(\frac{\Delta y}{\Delta x}\right) \\
& \theta=\tan ^{-1}\left(\frac{-\Delta z}{\sqrt{\Delta x^{2}+\Delta y^{2}}}\right)
\end{aligned}
$$

Here, the target is modelled as point object and the state variable form (Rogers, 2000) is used to represent the motion characteristics. For the purpose of simulation the initial detection of the target is taken as a random passive observation.

\section{Sensor model and formulation}

The RADAR and FLIR technical specifications for simulation can be taken from Fan et al. (2009) and Humali (2004). The measurement error for the RADAR is modelled after a Gaussian noise in which the variance in range and angle is given by (Keefer, 1989; Kondru and Celenk, 2018a, 2018b):

$$
\begin{gathered}
\sigma_{\text {range }}=\frac{\tau}{2.5 \sqrt{2 S N R}} \\
\sigma_{a z-e l}=\frac{\theta_{3 d b}}{2.5 \sqrt{2 S N R}}
\end{gathered}
$$

where SNR is given by:

$$
S N R=\frac{n P_{T} G_{T} G_{R} \sigma \lambda^{2}}{(4 \pi)^{3} k T B F\left(R_{\max }\right)^{4} L}
$$

The radar cross section (RCS) $\sigma$ is always fluctuating for non-stationary targets (Barton and Leonov, 1998). A physical optics approximation-based MATLAB tool known as POFACETS (Jenn, 2005) can be served as a convenient tool for RCS prediction. At the same time, the noises in the IR systems are categorised as distortions or statistical fluctuations in the electrical current such as Johnson noise, shot noise, generation-recombination noise and photon noises. Using the blackbody radiance theory and detector theory (Keefer, 1989) the total noise current is given by:

$$
\sigma_{\text {noise }} \text { or } n(R)_{\text {Photovoltaic }}=\sqrt{j n(r)+\operatorname{sn}(r)+p n(r)}
$$




\section{State variable theory and data fusion methods}

A popular finite-dimension or a state variable approach to represent state vector of a dynamic system is adopted in this paper. A generic representation (Jenn, 2015) for a given kinematic model is given by:

$$
\begin{aligned}
& \hat{X}_{k}(-)=F \hat{X}_{k-1}(+)+G w_{k-1} \\
& Z_{k}=H \hat{X}_{k-1}(+)+v_{k}
\end{aligned}
$$

Three different filters namely fixed-gain (Shimkin, 2009), Kalman (Grewal and Andrews, 2001) and particle filters (PFs) (LaViola, 2006; Wan and Van Der Merwe, 2000; Naidu, 2009) are studied and implemented for each target trajectory considered. Among the various multi sensor data fusion methods Kalman filter-based data fusion has been widely used (Wan and Van Der Merwe, 2000). In this section, instead of Kalman filter, EKF-based measurement fusion (MF) and state vector fusion (SVF) fusion algorithm is presented. The first step is to synchronise the data from both the sensors once at sampling period $\mathrm{T}$ of the tracking system. A centralised fusion tracking architecture is considered as shown in Figure 2.

Figure 2 Centralised fusion system block diagram

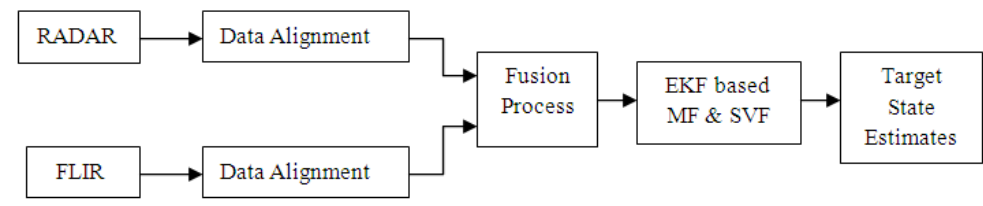

\section{Simulation analysis}

For the purpose of the analysis it is assumed both RADAR and FLIR are installed at the same location with the origin as their centre and the operating range is assumed to be $25 \mathrm{Km}$. The three degree of freedom in translation motion for different target motions are simulated in MATLAB with the assumed initial conditions. Each of the filter estimation process is done by 50 Monte Carlo simulations which is a relativistic way of obtaining possible outcomes. The performance of each estimator is ensured by computing the error analysis in positioning, range and angle accuracies using RMS errors. The principal function of these trackers is for better signal reconstruction by reducing measurement noise with less residual either in position or in angle. Now, the performance of each filter estimates is shown from Figures 3 to 6 . It is found that the efficiency of fixed gain filter is highly reliable upon the filter gain coefficients which are solely depend upon the smoothing coefficient that varies from zero to one. A value of 1 is chosen for heavy smoothing and low value for less smoothing. Whereas, the Kalman filter have better estimates than fixed gain filter because the Kalman gains are computed dynamically. In other words, the Kalman gain matrix determined from the state error variance as well as from measurement and noise variances adjusts adaptively. The Kalman filter computes its own state error uncertainty estimates while observing a new measurement. Also, as it is 
mentioned (Li et al., 2016), if the new measurement noise error variance is bigger than the state error variance, the Kalman filter will place less emphasis on the new measurement than if the state error were larger than the measurement error. On the other hand, the simple fixed gain filter computes the filter gains based on assigned smoothing coefficient. This effect and the superiority of Kalman filter over fixed gain estimator can be seen in position errors. Since the first target motion does not involve any nonlinearities fixed gain and Kalman filters serves as best linear estimators for reducing mean squared errors (MSEs). However, as all systems are ultimately nonlinear the high degree of nonlinearity associated with process and measurement equations makes a difficult state estimation problem for linear estimators like Kalman. On the other hand, this recursive Bayesian estimator known as PF provide better estimation accuracy for a non-Gaussian distribution. Here, the PF algorithm is based on estimating the probability distribution function (PDF) for the state variables given measurement variables and the generation of the particles is a trade-off. The key feature with PF over linear filtering is resampling which is to generate posteriori particles based on relative likelihood.

Figure 3 Sensor and filter estimates for projectile motion (see online version for colours)
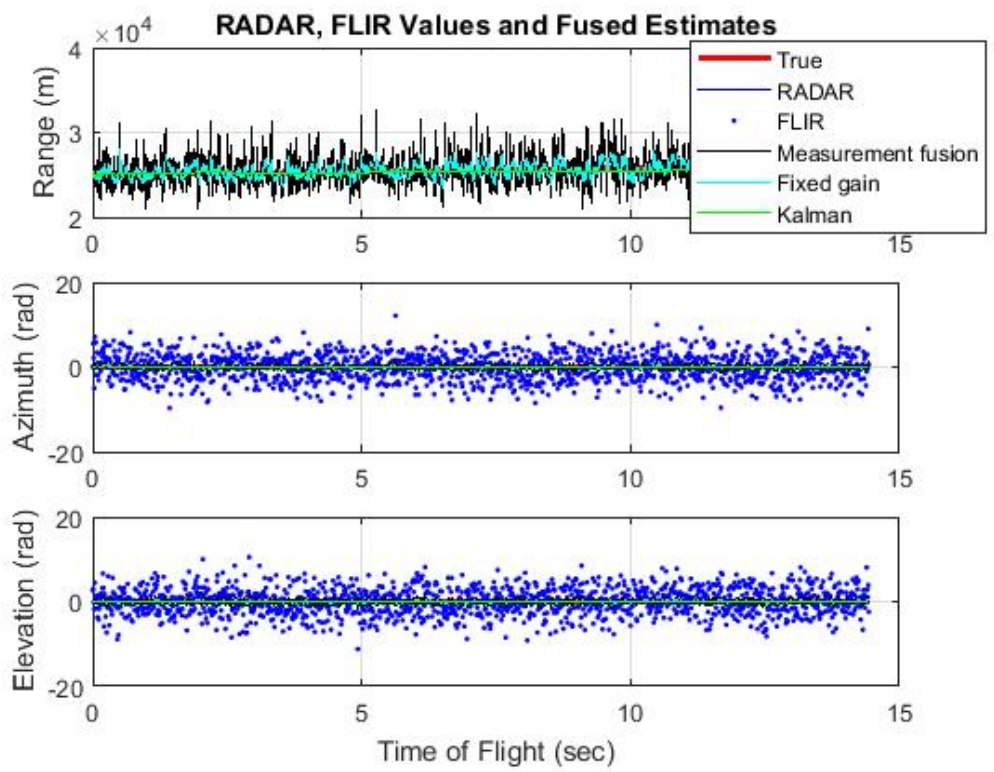

Figure 3 presents the important target features such as range, azimuth and elevation values by the sensor and the filter estimates. The comparison of RMS errors in target features is shown in Table 1. Among the entire filter estimates the MF and the SVF closely follows the true estimate giving the less RMS errors. Also, in Figure 4, the RMS error of a three dimensional target position is presented. The sensor or the measured value has the highest RMS error due to the inherent noises associated with radar. Although, fixed gain and the Kalman filter estimates produces less residual, the fusion algorithm produces much better result in reducing the RMS errors far better than individual filter estimates that proves the significance of fusion estimators. 
Figure 4 RMS errors for projectile motion (see online version for colours)

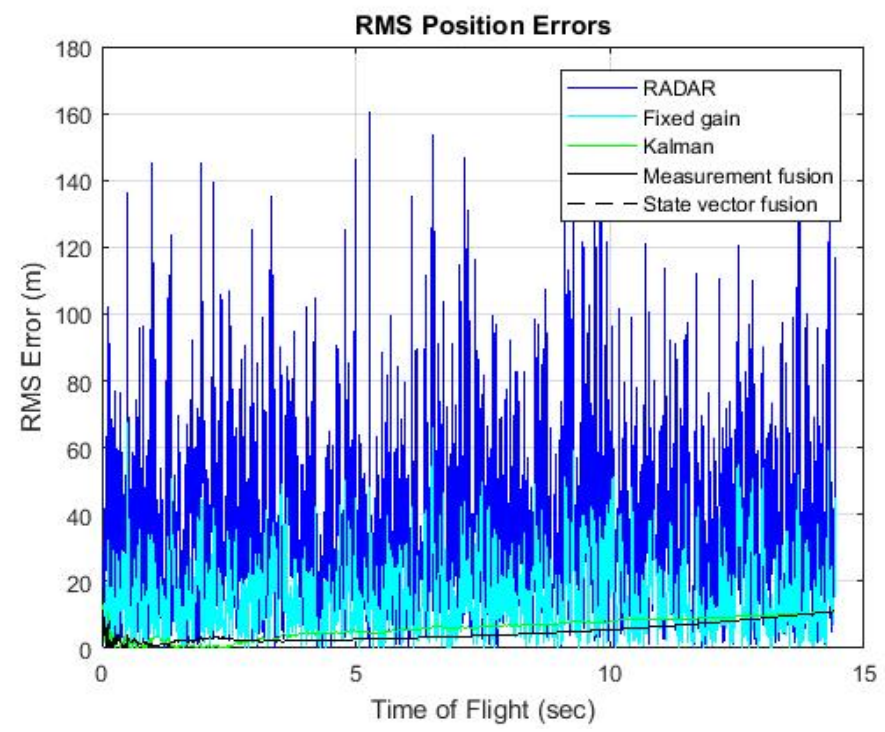

The MF and SVF methods are functionally equivalent with identical measurement matrices. Usually, SVF has less computational cost and is more efficient than MF. However, MF out performs SVF when the measurement matrices of the sensors are different. Also, it can be seen MF is more flexible especially when the measurement matrices and noise characteristics are time-varying. The RMS and RSSP errors in range and angles are shown in Table 2.

Figure 5 Sensor and filter estimates for nonlinear motion (see online version for colours)
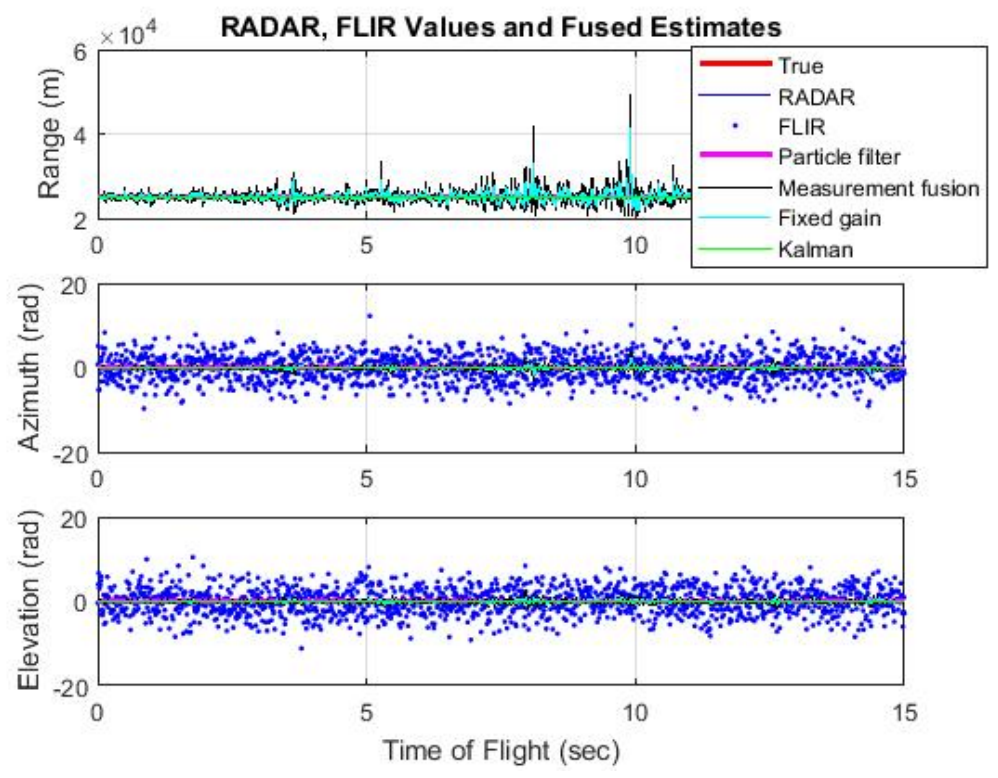
Figure 6 RMS errors for nonlinear motion (see online version for colours)

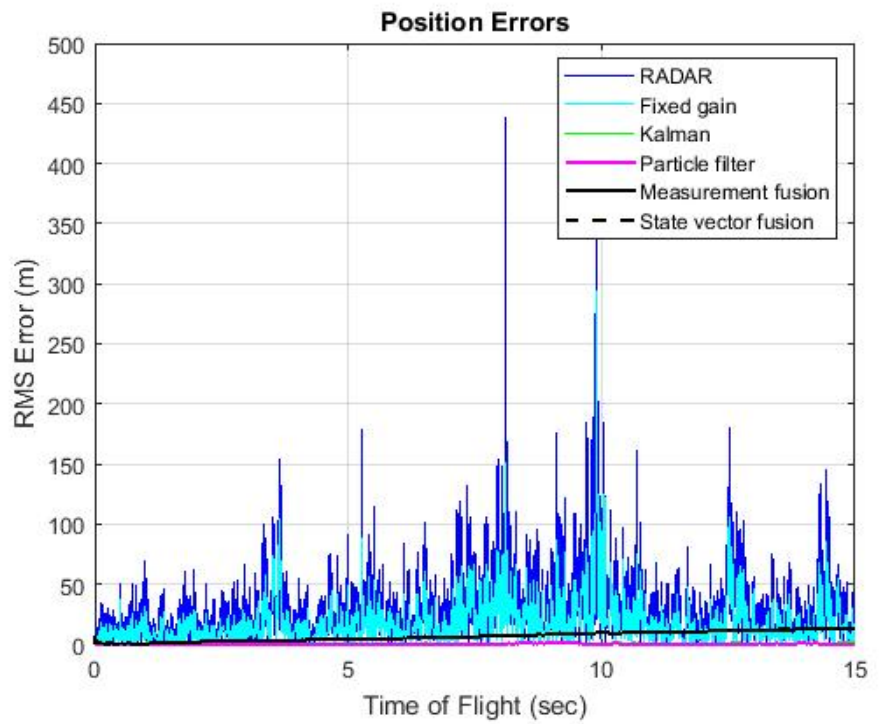

Table 1 Comparison of RMS and RSSP errors

\begin{tabular}{lcccc}
\hline \multirow{2}{*}{ Target motion } & Estimator & \multicolumn{3}{c}{ RMS errors } \\
\cline { 3 - 5 } Projectile & RADAR & 117.3 & $\psi(\mathrm{rad})$ & $\phi(\mathrm{rad})$ \\
& IRST & -- & 0.0021 & 0.0099 \\
& Fixed gain & 45.51 & 0.0136 & 0.0643 \\
& Kalman & 16.32 & 0.0082 & 0.0053 \\
& $M F$ & 11.11 & 0.0005 & 0.0002 \\
Nonlinear & 11.11 & 0.0024 & 0.0012 \\
& SVF & 29.98 & -- & -- \\
& RADAR & -- & 0.0027 & 0.0058 \\
& IRST & 24.57 & 0.0337 & 0.0735 \\
& Fixed gain & 17.65 & 0.005 & 0.0040 \\
& Kalman & 1.67 & 0.0006 & 0.0003 \\
& Particle & 13.53 & 0.0029 & 0.00005 \\
& MF & 13.78 & -- & 0.0063 \\
& SVF & & - \\
\hline
\end{tabular}

Figures 5 and 6 are the special case simulated for a nonlinear case given by the equation:

$$
x_{k}=0.5 x_{k-1}+\frac{25 x_{k-1}}{1+x_{k-1}^{2}}+8 \cos (1.2(k-1))
$$

where the target is aggressively manoeuvring in a nonlinear fashion. Here, $k$ is the discrete integer time index. For this special case, though the individual filter estimates and the fused estimators has performed better, $\mathrm{PF}$ has over ruled in providing better filter 
estimate and far less residual. In Figure 5, the PF is the closest filter estimate to the true value that proves its nature.

\section{Image fusion analysis}

Multi-imaging sensor fusion plays an important role for better enhancement of image for which the fused image will contain better contrast. As situational awareness has attained a greater importance, the image fusion process makes it easy for user to detect, recognise and identify the target. The wide variety of applications where image fusion applied is in medical imaging, microscopic imaging, remote sensing, computer vision and robotics. Based on the application, one of the main attributes while doing image fusion is to preserve the relevant data contained in the source images. It is absolutely necessary to remove the irrelevant features and noise to its maximum extent in the source image. Usually, the fusion process is carried out at pixel level by combining the source images without any pre-processing. Averaging of pixels of grey level images is one of the simplest kinds of multi-sensor image fusion. However, this simple fusion process results in undesirable effects and reduce the feature contrast. In some cases, various objects at different distances may not be captured by the imaging sensors in such a way that the object will be in focus than with the other sensor. In these scenarios, the conventional fusion process will fail.

Among the different fusion architectures, the source images are collected and combined as a whole for fusion. On the other hand, the source images are decomposed into small blocks to be used in fusion process. In the later case, the inconsistency is reduced as the local variations in the pixels are considered while fusing the images. The optimal size of the block and the threshold are set by the user while considering the spatial frequencies. An alternate method is a principal component analysis (PCA) method in which the source or the test images must be registered prior to the fusion process.

\subsection{PCA method}

The name of this method is principle component method that which transforms the number of correlated variables into a number of uncorrelated variables called principal components. The first principle component or the first uncorrelated variable will account for the highest variance in the data, and likewise each succeeding uncorrelated variable accounts for much of the remaining variance. The first principle component lies in the direction of maximum variance; the second principle component is constrained to lie in the subspace orthogonal to the first component for which this component within the subspace points to the direction of maximum variance. The third uncorrelated variable will lie in the direction of maximum variance of subspace orthogonal to the first two and it will continue likewise (Raol, 2009). Principal component basis vectors depend on the dataset. For instance, if $X$ be a d-dimensional random vector with zero mean, and orthogonal projection matrix $V$ be such that $Y=V^{T} X$. The covariance of $Y, \operatorname{cov}(Y)$ is a diagonal matrix. By applying simple matrix algebra: 


$$
\begin{aligned}
\operatorname{cov}(Y) & =E\left\{Y Y^{T}\right\} \\
& =E\left\{\left(V^{T} X\right)\left(V^{T} X\right)^{T}\right\} \\
& =E\left\{\left(V^{T} X\right)\left(X^{T} V\right)\right\} \\
& =V^{T} E\left\{X X^{T}\right\} V \\
& =V^{T} \operatorname{cov}(X) V
\end{aligned}
$$

Multiplying equation with $V$ on both sides:

$$
\begin{aligned}
V \operatorname{cov}(Y) & =V V^{T} \operatorname{cov}(X) Y \\
& =\operatorname{cov}(X) V
\end{aligned}
$$

The matrix $V$ can be written as $V=\left[V_{1}, V_{2}, \ldots, V_{d}\right]$ and $\operatorname{cov}(Y)$ in the diagonal form as:

$$
\left[\begin{array}{ccc}
\lambda_{1} & 0 & 0 \\
0 & \lambda_{d-1} & 0 \\
0 & 0 & \lambda_{d}
\end{array}\right]
$$

Using the above equation in:

$$
\left[\lambda_{1} V_{1}, \lambda_{2} V_{2}, \ldots, \lambda_{d} V_{d}\right]=\left[\operatorname{cov}(X) V_{1}, \operatorname{cov}(X) V_{2}, \ldots, \operatorname{cov}(X) V_{d}\right]
$$

The above equation could be written as:

$$
\lambda_{i} V_{i}=\operatorname{cov}(X) V_{i}
$$

where $i=1,2, \ldots, d$ and $V_{i}$ is an eigenvector of $\operatorname{cov}(X)$.

Here:

$$
I_{f k}=\left\{\begin{array}{c}
I_{1 k} N P C_{1 k}>N P C_{2 k}+t h \\
I_{2 k} N P C_{1 k}<N P C_{2 k}-t h \\
\frac{I_{1 k}+I_{2 k}}{2} \text { otherwise }
\end{array}\right.
$$

where $t h$ is the user defined threshold, and $\left(I_{1 k}+I_{2 k}\right) / 2$ is the average of corresponding gray level pixels.

\subsection{Experimental analysis}

To understand the advantages of PCA-based fusion method, the following images are considered for simulation. Two different types of noises are added to the images. One is Gaussian and the other is salt and pepper noise to see the performance of the PCA image fusion method. 
The image error measurements (Silva et al., xxxx; Gupta and Porwal, 2016; Wang and Bovik, 2002; Neto et al., 2013) are made based on the following definitions and equations:

1 MSE

The MSE between two images is given by:

$M S E=\frac{1}{M} \sum_{n=1}^{m} \sum_{m=1}^{n}[\hat{g}(n, m)-g(n, m)]^{2}$

where $M$ is the width of the image, $g(n, m)$ is the original image and $\hat{g}(n, m)$ is the estimated image. The disadvantage with the MSE is that it depends strongly on the image scaling. It varies from an 8-bit image to 10-bit image.

2 Root mean square error (RMSE)

The RMSE of an image is an absolute measure of it that measures the average magnitude of the error and is given by:

$$
R M S E=\sqrt{\frac{1}{M} \sum_{n=1}^{m} \sum_{m=1}^{n}[\hat{g}(n, m)-g(n, m)]^{2}}
$$

3 Peak signal to noise ratio (PSNR)

PSNR is a good measure for any restored image and avoids the problem of image scaling. For any image, PSNR is given by:

$P S N R=-10 \log _{10} \frac{M S E}{S^{2}}$

where $S$ is the maximum pixel value and PSNR is measured in $d B$. Although PSNR is common in use, it is not an ideal because the signal strength is estimated as $S^{2}$ rather than the signal strength of the actual image.

4 Mean absolute error (MAE)

MAE that calculates the average magnitude of the error is given by:

$$
M A E=\frac{1}{M N} \sum_{n=1}^{m} \sum_{m=1}^{n}|\hat{g}(n, m)-g(n, m)|
$$

5 Signal to noise ratio (SNR)

The SNR of an image determines the physical measure of the sensitivity of a digital imaging system. The industry standard of the SNR is given by:

$$
S N R=20 \log _{10} \frac{\text { signal }}{R M S \text { noise }} d B
$$

where the signal is the net difference between maximum and the minimum value of the image signal.

6 Image quality index (IQI) 
Another significant new development for the objective measure of an image is the IQI. The IQI definition is given by:

$$
Q=\frac{\sigma_{x y}}{\sigma_{x} \sigma_{y}} \cdot \frac{2 \overline{x y}}{(\bar{x})^{2}+(\bar{y})^{2}} \cdot \frac{2 \sigma_{x} \sigma_{y}}{\sigma_{x}^{2} \sigma_{y}^{2}}
$$

where the first term is known as linear correction, second term is luminance and the third term is known as contrast.

7 Enhancement measurement error (EME)

This is a measure of entropy derived to obtain optimal parameters for image enhancement. It is defined as:

$$
E M E_{r c}=\frac{1}{r \times c} \sum_{l=1}^{r} \sum_{k=1}^{c} 20 \ln \left[\frac{I_{\max ; k, l}}{I_{\min ; k, l}}\right]
$$

where $r \times c$ defines the blocks in which an image is split with being the size of the image.

8 Pearson correlation coefficient (PCC)

This is one of the widely used statistical techniques for pattern recognition and image processing where it computes the comparison of two images for image registration purpose, and disparity measurement. It is defined as:

$$
P C C=\frac{\sum_{i}\left(x_{i}-x_{m}\right)\left(y_{i}-y_{m}\right)}{\sqrt{\sum_{i}\left(x_{i}-x_{m}\right)^{2}} \sqrt{\sum_{i}\left(y_{i}-y_{m}\right)^{2}}}
$$

where $x_{i}$ is the intensity of the $i^{\text {th }}$ pixel in image $1, y_{i}$ is the intensity of the $i^{\text {th }}$ pixel in image $2, x_{m}$ is the mean intensity of image 1 , and $y_{m}$ is the mean intensity of image 2 . Table 2 presents the image error measurements for the images considered above.

Figure 7 Principle component analysis-based image fusion block diagram

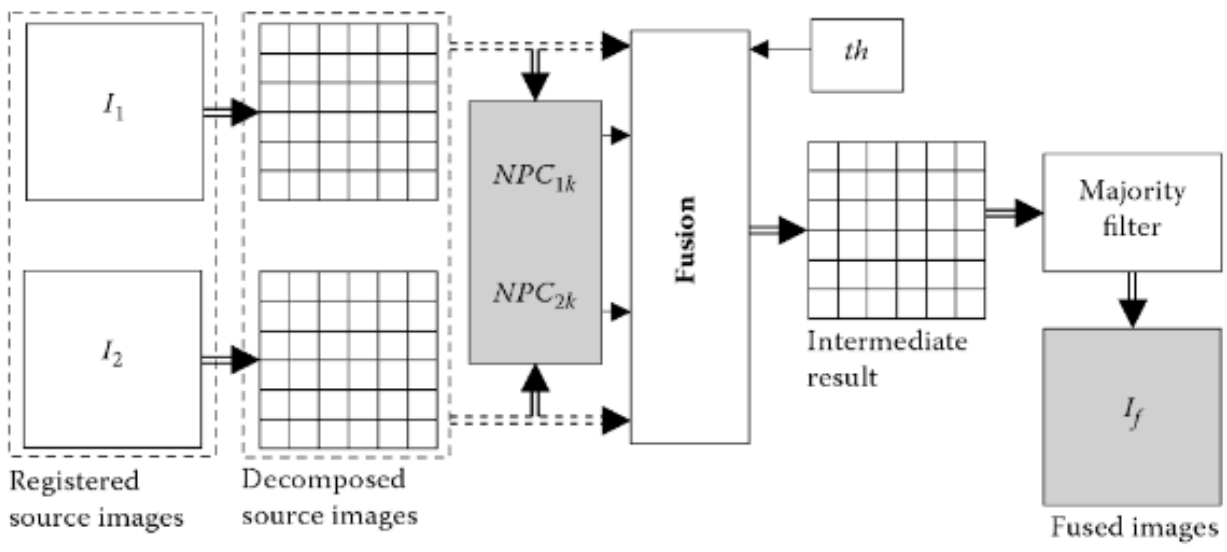

Source: Raol (2009) 
Figure 8 Original image and the noisy images
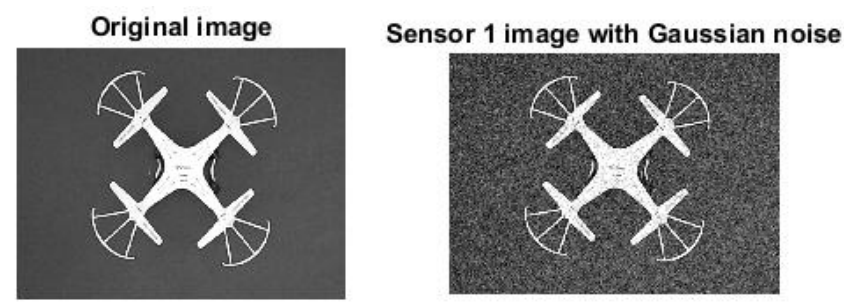

Sensor 2 image with salt \& pepper noise

PCA based fused image
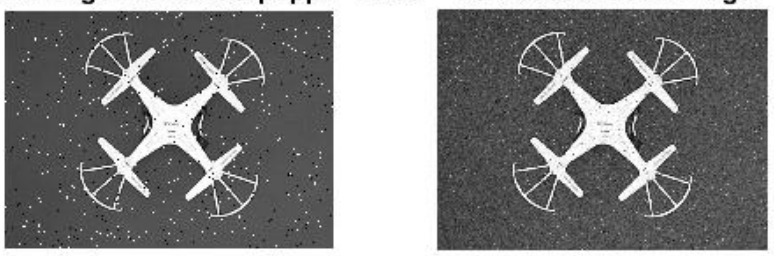

Figure 9 Error images
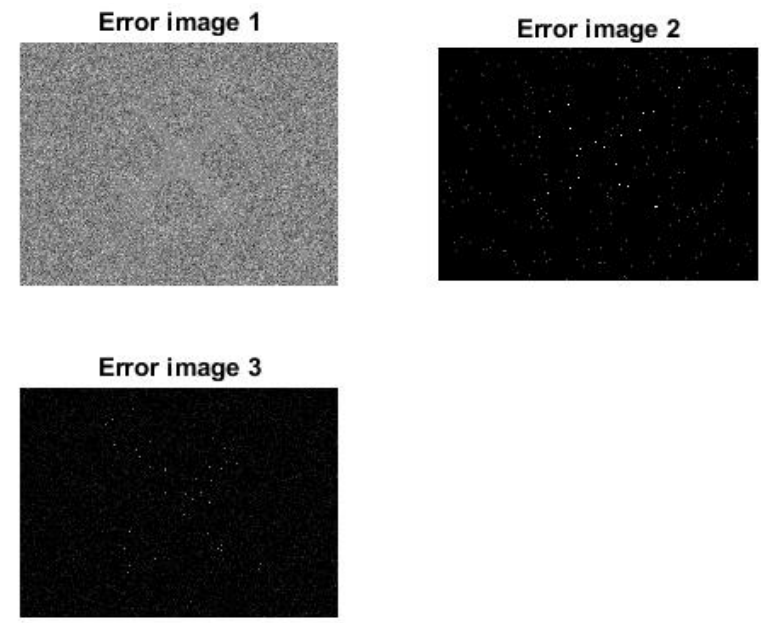

Table 2 Image measurement errors

\begin{tabular}{lccc}
\hline Image error & \multicolumn{3}{c}{ Image error measurements } \\
\cline { 2 - 4 } measurement & Sensor 1 image & Sensor 2 image & Fusion process \\
\hline RMSE & 0.0096 & 0.0061 & 0.0040 \\
PSNR & $68.36 \mathrm{~dB}$ & $70.30 \mathrm{~dB}$ & $72.19 \mathrm{~dB}$ \\
MAE & 0.07 & 0.01 & 0.04 \\
SNR & $13.5 \mathrm{~dB}$ & $13.6 \mathrm{~dB}$ & $14 \mathrm{~dB}$ \\
IQI & 0.05 & 0.33 & 0.07 \\
EME & 25.89 & 16.72 & 14.35 \\
PCC & $3.9 * 10^{6}$ & $4.09 * 10^{6}$ & $4.2 * 10^{6}$ \\
\hline
\end{tabular}




\section{Conclusions}

The mathematical modelling and formulation of target and sensor dynamics is utilised in this paper. A state variable approach is implemented for target, sensor and the filter tracking algorithms. By simulating different target motions, the performance evaluation of each filter is observed and the superiority of the multi sensor data fusion is also presented. A widely known image fusion technique known as principle component analysis (PCA) is also utilised to observe its robustness over single sensor performance. The RMS error measurement both in position as well as in image measurement error shows the necessity of multi sensor data fusion for better enhancement and improvement.

\section{References}

Barton, D.K. and Leonov, S.A. (1998) Radar Technology Encyclopedia, Artech House, Inc., Cambridge, MA, USA.

CommandSpace Cereberus Datasheet (2018) 2015 FLIR Systems Inc., 21 May [online] http://www.FLIR.com (accessed 20 March 2018).

Dimitrios, P. (2007) Track Score Processing of Multiple Dissimilar Sensors, Master thesis, June, Dept. of Information Sciences, Naval Postgraduate School Monterey, California.

Fabrizio, A. (2014) 'Advances in target detection and tracking in forward-looking InfraRed (FLIR) imagery', Sensors-MDPI, Vol. 14, pp.20297-20303, ISSN 1424-8220.

Fan, L., Wang, J. and Shu, D. (2009) 'Detection of a dim point target using dynamic programming approach', Progress In Electromagnetics Research Symposium, Beijing, China, 23-27 March.

FLIR The World's Sixth Sense (2016) Discover the best Technology for Border Surveillance, FLIR Systems, Inc.

Friedman, N. (2009) Network Centric Warfare, 1 March, US Naval Institute Press, NY City, USA.

Garwin, R.L. (1999) Technical Aspects of Ballistic Missile Defense, Arms Control and National Security Session, APS, Atlanta, March.

Gordon, N.J., Salmond, D.J. and Smith, A.F.N. (1993) 'Novel approach to nonlinear/non-Gaussian Bayesian state estimation', IEE Proceedings- $F$, April, Vol. 140, No. 2, pp.107-113.

Grewal, M.S. and Andrews, A.P. (2001) Kalman Filtering Theory and Practice using MATLAB, 2nd ed., Wiley \& Sons, Inc., NY City, USA.

Gupta, S. and Porwal, R. (2016) 'Appropriate contrast enhancement measures for brain and breast cancer images', International Journal of Biomedical Imaging, Article ID 4710842, 8pp.

Humali, I.G. (2004) Sensor Fusion for Boost Phase Interception of Ballistic Missiles, Master thesis, September, Dept. of Information Sciences, Naval Postgraduate School Monterey, California.

Jenn, D.C. (2005) Radar and Laser Cross Section Engineering, 2nd ed., 20 August, AIAA Education Series, California, USA.

Jenn, D.C. (2015) POFACETS 4.3 - Radar Cross Section (RCS) Prediction Code Based on the Physical Optics (PO) Approximation, 23 April [online] https://www.mathworks.com/ matlabcentral/fileexchange/50602-pofacets4-3.

Keefer, C.W. (1989) Infrared Target Detection: Signal and Noise Sensitivity Analysis, Master thesis, December, School of Engineering of the Air Force Institute of Technology, Wright-Patterson Air Force Base, Ohio.

Khalifa, N.S. (2013) 'Space base laser torque applied on LEO satellites of various geometries at satellite's closest approach', NRIAG Journal of Astronomy and Geophysics, Vol. 2, No. 2, pp.266-271. 
Kondru, D. and Celenk, M. (2018a) 'Predictive airborne target tracking using all-terrain fusion based mobile surveillance system', Proceedings 52nd Annual IEEE Conference on Information Sciences and Systems, Princeton University, 21-23 March.

Kondru, D. and Celenk, M. (2018b) 'Mitigation of target tracking errors and sUAS threat response using multi sensor data fusion', EURASIP IAPR - 2018 International Conference on Image and Signal Processing, University of Normandy, France, 2-4 July.

Landsat 8 (2013), NASA Landsat Science, 11 February [online] https://landsat.gsfc.nasa.gov/landsat-8/ (accessed April 2018).

LaViola, J.J. (2006) 'A comparison of unscented and extended Kalman filtering for estimating quaternion motion', Proceedings of the American Control Conference, June.

Lesson 2 - Projectile Motion, Projectile Motion and Inertia, The Physics Classroom, 1996-2017 [online] http://www.physicsclassroom.com/Class/vectors/u312a.cfm (accessed 3 April 2018).

Li, B., Ren, G., Pan, Z. and Tingting, T. (2016) 'Moving target detection and tracking interactive algorithm based on acoustic image', 2016 IEEE Conference on Ocean Acoustics (COA), Harbin, China.

Mahafza, B.R. (2000) Radar Systems Analysis and Design Using MATLAB, Chapman \& Hall/CRC, Corporate Blvd., Boca Raton, Florida.

Minvielle, P. (2005) 'Decades of improvements in re-entry ballistic vehicle tracking', IEEE Aerospace and Electronic Systems Magazine, August, Vol. 20, No. 8, pp.386-438.

Naidu, V.P.S. (2009) 'Fusion of radar and IRST sensor measurements for 3D target tracking using extended Kalman filter', Defence Science Journal, March, Vol. 59, No. 2, pp.175-182.

Neto, A.M., Victorino, M.C., Fantoni, I., Zampieri, D.E., Ferreira, J.V. et al. (2013) 'Image processing using pearson's correlation coefficient: applications on autonomous robotics', 13th International Conference on Mobile Robots and Competitions (Robotica 2013), Lisbon, Portugal, April, pp.14-19.

Nykamp D.Q., Spherical Coordinates, Math Insight [online] http://mathinsight.org/ spherical_coordinates (accessed 3 April 2018).

Raol, J.R. (2009) Multi-Sensor Data Fusion with MATLAB, 1st ed., 26 December 16, CRC Press, Cambridge.

Rodríguez-Canosa, G., del Cerro Giner, J. and Barrientos, A. (2014) 'Detection and tracking of dynamic objects by using a multirobot system: application to critical infrastructures surveillance', Sensors Journal - MDPI, 12 February.

Rogers, R.M. (2000) Applied Mathematics in Integrated Navigation Systems, July, AIAA Education Series, Boston, MA.

Shimkin, N. (2009) Estimation and Identification in Dynamical Systems, Lecture Notes, Fall, Department of Electrical Engineering, Technion-Israel Institute of Technology.

Silva, E.A., Panetta, K. and Agaian, S.S., Quantifying Image Similarity using Measure of Enhancement by Entropy [online] http://ww.ece.tufts.edu/ esilva02 (accessed 3 April 2018).

Wan, E.A. and Van Der Merwe, R. (2000) 'The unscented Kalman filter for nonlinear estimation', Proceedings of the Adaptive Systems for Signal Processing, Communications, and Control Symposium, October.

Wang, Z. and Bovik, A.C. (2002) 'A universal image quality index', IEEE Signal Processing Letters, Vol. 9, No. 3, pp.81-84.

Weisstein, E.W., Helix, MathWorld - A Wolfram Web Resource [online] http://mathworld. wolfram.com/Helix.html (accessed 3 April 2018). 